\title{
Morphological changes during annealing of polyethylene nanocrystals
}

\author{
N. Basu ${ }^{1, a}$, A. Osichow ${ }^{2}$, S. Mecking ${ }^{2}$, and G. Reiter ${ }^{1}$ \\ 1 Physikalisches Institut, Albert-Ludwigs Universität Freiburg, Freiburg, Germany \\ 2 Fachbereich Chemie, Universität Konstanz, Konstanz, Germany
}

\begin{abstract}
Polymer crystals are metastable and exhibit morphological changes when being annealed. To observe morphological changes on molecular scales we started from small nanometer-sized crystals of highly folded long-chain polymers. Micron-sized stripes consisting of monolayers or stacks of several layers of flat-on oriented polyethylene nanocrystals were generated via evaporative dewetting from an aqueous dispersion. We followed the morphological changes in time and at progressively higher annealing temperatures by determining the topography and viscoelastic properties of such assemblies of nanocrystals using atomic force microscopy. Due to smallness and high surface-to-volume ratio of the nanocrystals, already at $75{ }^{\circ} \mathrm{C}$, i.e. about 60 degrees below the nominal melting point, the lateral size of the crystal coarsened. Intriguingly, this occurred without a noticeable reduction in the number of folds per polymer chain. Starting at around $110{ }^{\circ} \mathrm{C}$, chain folds were progressively removed leading to crystal thickening. At higher temperatures, but still below the melting point, prolonged annealing allowed for surface diffusion of molten polymers on the initially bare substrate, leading eventually to the disappearance of crystals. We compared these results to the behavior of the same nanocrystals annealed in an aqueous dispersion and to bulk samples.
\end{abstract}

\section{Introduction}

Nanoparticles having a crystalline structure, and thus distinct molecular order and orientation, have been used as functional units and building blocks for larger scale, mesostructured two- and three-dimensional objects (like stripes, films of discrete thickness, stacks of layers, ...) [13]. However, mainly inorganic particles have been studied because it has been difficult to synthesize polymoric crystals of such small size which also can be processed further. While single polymer single crystals have been obtained by crystallization from highly dilute solutions [4,5], the amount of crystalline material, which was obtained under such conditions, was typically extremely low. Fortunately, an alternative synthetic approach for the formation of polymeric nanocrystals was developed recently. By using water-soluble $\mathrm{Ni}$ (II)-complexes as catalysts, aqueous dispersions containing well-defined and characterized polyethylene nanocrystals have been obtained in large numbers [6-8]. These well-separated and easily processable nanocrystals open up new routes for functional structure formation at various lengthscales [9]. In addition, these nanocrystals can be functionalized by synthetic modification, e.g. via endgroups or via the integration of functional groups at regular position along the polymer

\footnotetext{
${ }^{\text {a }}$ e-mail: nandita.basu@physik.uni-freiburg.de
}

chain using acyclic diene metathesis polymerization (ADMET) $[10,11]$. Unfortunately, polymeric crystals are often highly metastable. Even at temperatures below the melting point, such crystals may change shape, size and morphology in the course of time. Thus, in order to successfully implement such nanocrystals into functional devices, we need a detailed understanding of their stability and morphological evolution at various temperatures.

Given that we have sufficient control over metastability of polymer nanocrystals, the use of pre-fabricated building blocks with molecular order provides the possibility for advanced molecular ordering on length scales much larger than the size of the particle $[12,13]$. Following appropriate ways of assembly, nanocrystals can be arranged in regular patterns [14-16]. Moreover, using anisotropic nanoparticles, these patterns may also exhibit specific, directiondependent properties given that a unique orientation of the building blocks was achieved $[17,18]$. To reach such goal, structure formation guided through solvent evaporation has been studied previously $[19,20]$. Special attention of theoretical and experimental studies has been focused on the behavior in the vicinity of a three phase contact line [19-21], There, hydrodynamic transport of nonvolatile solutes towards the contact line is combined with solvent evaporation. Often the resulting patterns are periodic $[19$, $20,12,13]$. Evaporation from a drop containing solid particles typically results in the formation of ring-like patterns 
of precipitate. This phenomenon is related to the so-called coffee stain effect [22-25]. As maximum evaporative loss takes place at the perimeter of the drop [22], all the solutes are transported to the edge by an outward hydrodynamic flow. Jamming of the solutes [26] at the perimeter caused a local but transient pinning of the contact line.

Because of its mechanical properties and low cost of production polyethylene (PE) is extensively studied and widely used in many applications. Due to its simple chemical structure, PE also represents one of the most investigated polymers in terms of crystallization in solution, bulk systems or thin films [27-33]. In general, in order to proceed from a disordered polymer melt to a perfectly ordered crystal significant changes in conformations are required [34]. However, in particular the length of the polymer imposes severe steric and kinetic restrictions that result in crystalline states having some degree of disorder like chain folds [35]. For a given polymer of known length, a measure for the degree of folding is the thickness of the crystalline lamellae: the thinner these lamellae are, the more folded are the polymer chains. The number of chain folds, and thus the thickness of the lamellae, highly depends on crystallization conditions. For long chains, the equilibrium state, represented by a lamellar crystal consisting of fully extended chains [36], is never reached.

It turns out that due to the synthesis, which has to be performed at low temperatures, the resulting polyethylene nanocrystals contain highly folded polymer chains which thus are far from their thermodynamic equilibrium state $[8,9]$. Consequently, these nanocrystals will unavoidably change their morphology even when kept at temperatures far below their nominal melting point. For such highly metastable states of small polymer crystals, it is a nontrivial task to define and measure the melting process. Like for small inorganic crystals or clusters, an individual crystal may melt at a lower temperature than the bulk melting temperature $[37,38]$ : The melting temperature decreases as the surface-to-volume ratio of the crystal increases. In addition, polymer crystals consisting of folded molecules are able to increase their crystallinity by a reduction of the degree of chain folding with temperature or time. In most cases, molecular re-organization into less folded states improves the thermal stability of the crystal and shifts its melting temperature to higher values. In the presence of neighboring small nano-crystals, molecules maybe exchanged between these crystals. Such exchange may lead to coarsening, i.e. slightly bigger crystals may become larger at the expense of smaller crystals $[39,40]$. Due to their lower surface-to-volume ratio, bigger crystals typically have a higher melting temperature than smaller ones. Recently, computer simulation results were compared to experiments on alkane chains and functional olefins validating the inverse thickness dependence of the melting temperature (Gibbs-Thompson behavior) which also was influenced by the crystal environment, i.e. interfacial tensions [41]. Consequently, instead of melting, crystals may coarsen and thicken and so increase their melting temperature.

Here, we will explore the consequences of metastability of polymer crystals by detecting changes in morphology as a result of annealing. Using well-defined nanometer-sized polyethylene crystals consisting of highly folded long-chain polymers, we followed these changes as a function of temperature and annealing time.

\section{Experimental procedures}

\subsection{Polyethylene nanocrystal dispersions}

Aqueous dispersions with a solid content of $\sim 3.5 \mathrm{wt} \%$ of nanocrystals of polyethylene having a molecular weight $M_{n} \sim 2 \cdot 10^{5} \mathrm{~g} / \mathrm{mol}$ (ca. $10^{4}$ monomers) and a polydispersity index $M_{w} / M_{n} \sim 2$ were chosen for our studies. The nanocrystals were prepared by catalytic polymerization of ethylene with water soluble $\mathrm{Ni}(\mathrm{II})$ complexes at $\mathrm{T}=15^{\circ} \mathrm{C}$ and a pressure of $4 \mathrm{MPa}$, causing a high degree of supercooling [8]. The resulting polymers have approximately 5 methyl-branches per 1000 carbon atoms in the backbone of the chain. Sodium dodecyl sulfate (SDS) was employed to stabilize the nanocrystals against coagulation. The surface tension of the dispersion was found to be relatively high $(\sim 58 \mathrm{mN} / \mathrm{m})$ indicating that virtually all SDS surfactant molecules were adsorbed onto the nanocrystals, i.e. almost no free SDS molecules or micelles were present in the system. We note that the resulting morphologies after annealing described below did not change qualitatively after removing free SDS molecules by washing the deposited layers of nanocrystals in pure water. Only the rate of these changes increased somewhat after removing SDS.

As shown previously by using cryo-TEM and in combination with small-angle X-ray scattering [8] these dispersions contained essentially well-separated, nano-sized platelet-like crystals (lateral size $\sim 25 \mathrm{~nm}$, thickness $\sim$ $8 \mathrm{~nm}$ ) consisting of a single crystalline lamella (thickness $\sim 6 \mathrm{~nm}$ ) covered by thin amorphous layers (thickness $\sim 1 \mathrm{~nm}$ ). These crystals exhibited a crystallinity of ca. $70 \%$ [8] and a melting temperature of ca. $133^{\circ} \mathrm{C}$, as determined by differential scanning calorimetry (DSC) at a rate of $10 \mathrm{~K} / \mathrm{min}$. We note that this temperature is still below the theoretical equilibrium melting point of polyethylene $\left(141^{\circ} \mathrm{C}\right)[42,43]$. For the studies described below, we used two concentrations of the aqueous dispersion $35 \mathrm{ppm}$ and $70 \mathrm{ppm}$ by adding pure water in proper amount to the stock solution of $0.35 \mathrm{wt} \%$.

\subsection{Deposition technique}

In order to be able to employ microscopy for the investigation of molecular properties of polymer nanocrystals we had to deposit the crystals in a controlled and ordered way. Starting from a defined initial organization of nanocrystals allows to relate thermally induced morphological changes (e.g. increase in lamellar thickness or lateral size of crystalline regions) with reorganization processes occurring on a molecular scale. To this end and due to its simplicity, an evaporation-based deposition process was chosen which resulted in patterns of controllable size and containing only 

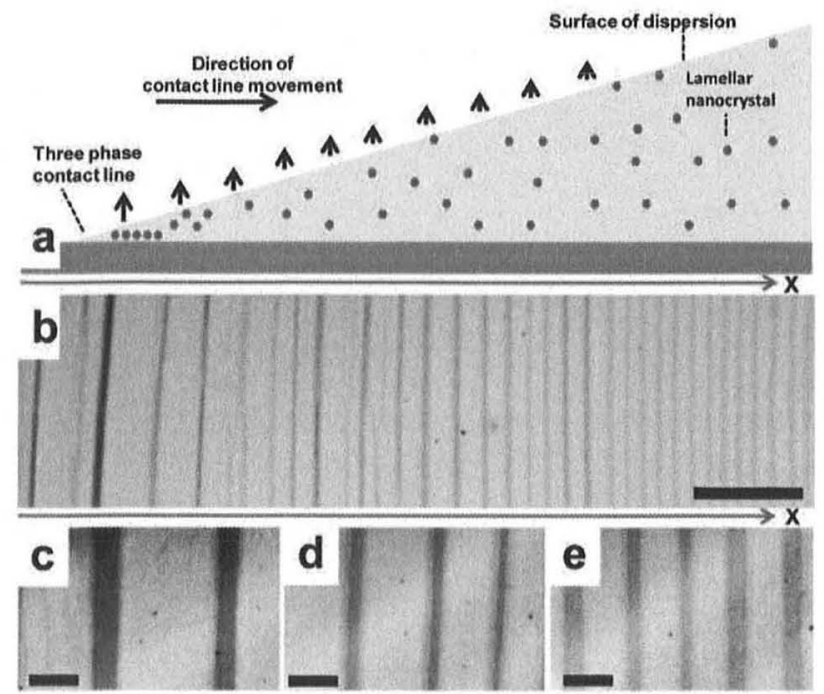

Fig. 1. (a) Schematic representation of the retracting threephase contact line in the course of evaporative dewetting of an aqueous dispersion of polyethylene nanocrystal. Experimentally, the set up consisted of a $1 \mathrm{~cm}$ inner-diameter Teflon ring on top of a silicon substrate filled with the aqueous dispersion. The $x$-axis indicates the direction of dewetting, i.e. from the center towards the bounding Teflon ring. (b) Optical micrograph of a small slice (ca. $200 \times 700 \mu \mathrm{m}^{2}$ ) at a distance of ca. $2 \mathrm{~mm}$ from the center, taken after complete evaporation of water. (c-e) Higher-resolution optical micrographs showing the typical changes as the dewetting processes moves from a position about $2 \mathrm{~mm}$ from the center (c) toward the Teflon ring $(\mathrm{d}+\mathrm{e})$. The scale bar in images (b) and (c-e) represents $100 \mu \mathrm{m}$ and $10 \mu \mathrm{m}$, respectively.

flat-on oriented nanocrystals. Imposing a circular geometry by means of a Teflon ring (inner diameter of $1 \mathrm{~cm}$ ), we have placed a small volume (ca. $0.2 \mathrm{ml}$ ) of the aqueous nanocrystal dispersion onto a clean silicon substrate. The whole set-up was kept at room temperature and left open to air, allowing for evaporation of water. Typically, the evaporation processes was completed within 30-60 min. Capillary forces acting within the Teflon ring generated a long-range liquid meniscus. In the course of evaporation, a dry spot bounded by circular three-phase contact line was formed close to the center of the ring where the liquid film of the aqueous dispersion was thinnest. This dry spot induced dewetting (see fig. 1a) in the radial outward direction. The underlying basic process has been named "evaporative dewetting" $[16,44,45]$. By controlling the evaporation rate (e.g., via temperature and/or vapor pressure) the dewetting velocity could be varied. A similar approach was used for the organization of spherical gold nanoparticles. That study focused in great detail on the role of evaporation rate and contact line dynamics [16]. Guided through evaporative dewetting, it was possible to produce ordered patterns in a simple and controllable manner. In contrast, thin films of dispersion produced by spincoating exhibited fast dewetting and resulted in a broad range of irregular patterns on the surface $[15,46-48]$.
The presence of nanocrystals led to repeated transient pinning, and thus to a non-monotonic displacement of the retracting contact line. This stick-slip process resulted in the formation of micrometer-wide concentric annuluses. On a scale much smaller than the diameter of these annuluses the deposited nanocrystals appear as parallel stripes. The formation of stripe patterns has been discussed in theoretical models $[21,49]$. Within the aqueous dispersion, polyethylene nanocrystals were transported by convective flow towards the contact line where they got stuck, locally jammed and formed a clog. The so localized nanocrystals exerted a frictional or pinning force which slowed down dramatically or even stopped the motion of the contact line. During pinning the contact area between dispersion and substrate remained constant. As a consequence, the loss of volume due to evaporation caused a steady decrease of the contact angle. In turn, the uncompensated Young force [50] increased persistently until it overcame the pinning or frictional force. This led to depinning of the contact line, which jumped to a new position where it got arrested once more. The deposited polyethylene nanocrystals were rather strongly adsorbed on the silicon substrate as can be demonstrated by their resistance to desorption even when the whole sample was put in large volumes of pure water. The stripes remained almost unchanged after such a washing procedure.

At a distance of ca. $2 \mathrm{~mm}$ from the center of the Teflon ring, a small slice $\left(\right.$ ca. $200 \times 700 \mu \mathrm{m}^{2}$ ) of the whole sample was observed with an optical microscope. Exploiting interference colors of light reflected from the substrate and from the surface of the deposited structures, we were able to detect a series of parallel stripes of nanometric thickness by optical microscopy. These stripes represent segments of the annuluses formed during evaporative dewetting (see fig. 1b). As confirmed by atomic force microscopy (AFM), the various gray levels seen in the micrograph (fig. 1b) can be directly related to the thickness of the stripe: The darker the gray the thicker the stripe. Close to the center of the Teflon ring, i.e. the position where the dewetting process started, stripes were spaced at rather irregular distances (see fig. 1c). As dewetting evolved, more regularly spaced stripes (see fig. $1 \mathrm{~d}+\mathrm{e}$ ) were generated. We assume that the progressive evaporation of water led to a gradual change in number density of PE nanocrystals in the dispersion which, in turn, caused more frequent pinning of the contact line.

Using AFM, it could be shown that the deposited stripes had a well-defined thickness. Especially towards the end of the evaporation process, where closely spaced stripes were observed (fig. 1e), they all had a uniform thickness of about $8 \pm 1 \mathrm{~nm}$ (fig. $2 \mathrm{a}, \mathrm{b}+\mathrm{c}$ ). This thickness is practically identical with the lamellar thickness of the polyethylene nanocrystals, as determined by X-ray scattering and electron microcopy $[8,9]$. This uniform thickness suggests that the stripes were formed by a single layer of flat-on oriented nanocrystals crystals, i.e. crystals having their lamellar normal in the direction of the surface normal. As shown previously [51], flat-on PE lamellae most likely contain polyethylene chains with the $c$-axis of 

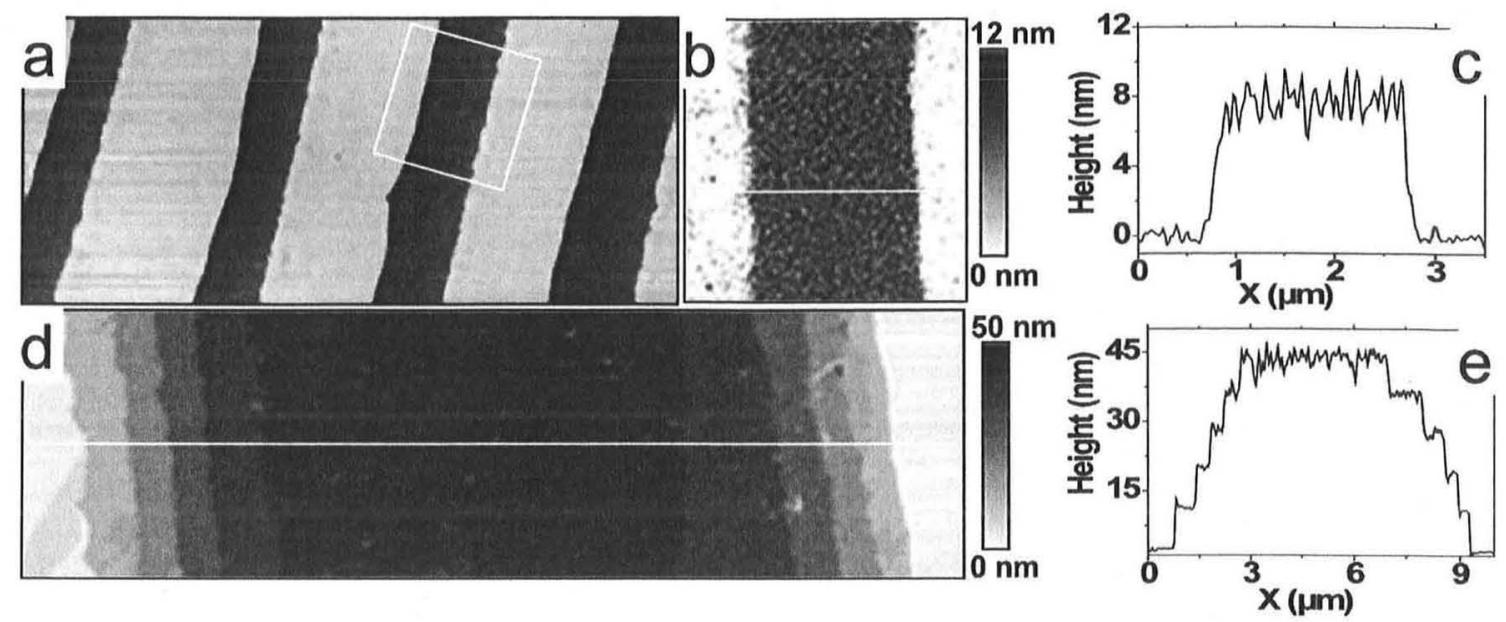

Fig. 2. (a) AFM topography image $\left(28 \times 13 \mu \mathrm{m}^{2}\right)$ of regularly spaced stripes obtained towards the end of the evaporative dewetting process (similar to fig. 1e). It should be noted that all stripes exhibited a unique mean thickness as can be deduced from the color code. b) Higher resolution image $\left(3 \times 3 \mu \mathrm{m}^{2}\right)$ and c) a cross-section through one of these stripes. d) AFM topography image $\left(12 \times 3.2 \mu \mathrm{m}^{2}\right)$ stack of five layers obtained at an earlier stage of the evaporative dewetting process (similar to fig. 1c). e) Cross-section through this stack. Note that the mean height of the monolayer (ca. $8 \mathrm{~nm}$ ) is equal to the step height in the stack.

the unit cell (i.e. the PE-stem) oriented at an angle of ca. $35^{\circ}$ to the direction of the surface normal.

The formation of stacks of several layers (fig. $2 \mathrm{~d}+\mathrm{e}$ ) was observed at early stages of the evaporation process, or when more concentrated dispersions were used. Interestingly, step heights were similar for monolayers and each step of multiple layers (fig. 2). From characteristic height profiles (fig. $2 \mathrm{c}+$ e) we deduced a regular step height of $8 \pm 1 \mathrm{~nm}$ for these steps. Thus, we concluded that all nanocrystals were oriented flat-on, i.e. as lamellae parallel to the substrate surface.

Complementary experiments showed that the width of stripes and their spacing can be influenced by the initial concentration of the dispersion, the rate of evaporation of water and the solvent-substrate interaction. For the purpose of studying the influence of annealing on morphological changes of the nanocrystals, we mainly focused on regular monolayer and multilayer stripes of up to some micrometers in width.

\subsection{Microscopy tools}

Real time studies of the dewetting process induced by evaporation of the solvent (water), the stick-slip motion of the contact line and the corresponding deposition of stripes of crystals were viewed through an optical microscope in reflection mode (OLYMPUS BX $51 \mathrm{M}$ ). After complete evaporation, these stripes were characterized in detail using an atomic force microscope (AFM) in the tapping mode (JPK-Nanowizard). PPP-NCL-W tips (NANOSENSORS) with force constant ranging between 21 and $98 \mathrm{~N} / \mathrm{m}$ were used as AFM probes. Using a hot stage allowed us to visualize in situ reorganization and fusion of the deposited nanocrystals in real time at various annealing temperatures.

\section{Results and discussion}

A polymer crystal containing folded chains does not represent the lowest free energy state and thus is only metastable. Such folded states of reduced degree of crystalline order are prone to morphological changes which are accompanied by a reduction of the number of folds per molecule. A process of morphogenesis [52] can be identified, following characteristic paths which lead to a reduction of the total free energy. The process can be modeled by computer simulation [53] considering individual chains as the basic units which have the possibility of internal reorganization, even behind the growth front, i.e. in a crystalline environment. As indicated by these computer simulations, the process of morphogenesis is more pronounced for small crystals of initially highly folded polymer chains. The here investigated nanocrystals consist on the average of eight chains with several hundred folds per molecule. This high degree of folding of the polymers is the consequence of the low temperature (large undercooling) at the stage of polymerization and nanocrystal formation. Consequently, these nanocrystals are highly metastable and therefore will exhibit a strong tendency to change their morphology. In the present study, we followed the pathways taken during morphogenesis through in situ AFMstudies on monolayer stripes of these nanocrystals.

The limited number of polymer chains per nanocrystal and their well-defined orientation with respect to the substrate facilitate to follow and to relate the observed morphological changes to molecular transport and conformational changes (reduction of chain folds). The high surface-to-volume ratio, i.e. the large number of molecules located at the periphery of the crystal with respect to the interior, translates directly into a high detachment probability for polymers from the nanocrystal and thus favors rapid morphological changes. 
In the following, we will present our results as a function of increasing annealing temperature which enables progressively faster and more long-ranged morphological changes. We will also compare observations on monolayers with the simultaneously occurring changes in multilayers.

\subsection{Annealing experiments at $\mathrm{T}<110^{\circ} \mathrm{C}$}

Monolayer stripes obtained by the here employed evaporative dewetting process are built up from a random assembly of nanocrystals which are neither all uniquely oriented nor perfectly ordered without having empty space in between. Accordingly, due to such boundaries within the monolayer, many polymer molecules (or parts of them) are located at crystal surfaces where they are lacking neighboring polymers. Consequently, as temperature increases, these molecules have a higher probability for detaching from the crystal and becoming part of a molten phase surrounding the nanocrystals. Using tapping mode AFM the amount of molten polymers can be visualized via the phase-signal.

In fig. 3 , we observed the morphological changes occurring in a monolayer stripe during annealing at a comparatively low temperature where according to DSC the nanocrystals do not melt. We have chosen $75^{\circ} \mathrm{C}$, i.e. almost $60^{\circ} \mathrm{C}$ below the nominal melting temperature of $133^{\circ} \mathrm{C}$. Similar observations of morphological changes, though at varying rates, were made at all temperatures in between ca. $60^{\circ} \mathrm{C}$ and $110^{\circ} \mathrm{C}$. As can be seen from fig. $3 \mathrm{a}^{\prime}$, the as-prepared sample showed already some heterogeneities indicating the random assembly of nanocrystals which did not results in a highly ordered pattern. The corresponding phase image (fig. 3a" $\mathrm{a}^{\prime \prime}$ ), indicates that the whole stripe consisted of highly elastic material similar to the surrounding substrate, as represented by the bright color of the phase signal. No molten regions were detectable. However, when increasing the temperature to $75^{\circ} \mathrm{C}$ the phase signal changed significantly (fig. $3 \mathrm{~b}^{\prime \prime}$ ), indicating that some molecules were molten. Even after prolonged annealing, the phase contrast did not change much (fig. $3 \mathrm{c}^{\prime \prime}$ ). Alongside with the change in phase contrast, an apparent change in thickness (height) of the stripe was observed (fig. $3 b^{\prime}+c^{\prime}$ ) [54]. When pushing the AFM tip harder into the sample, i.e. penetrating through a layer of molten polymers on top of the stripe, the heterogeneous arrangement of fused crystals became visible in topography (fig. $3 \mathrm{~d}^{\prime}$ ). The corresponding phase image (fig. $3 \mathrm{~d}^{\prime \prime}$ ) shows that between the nanocrystals a significant number of molten polymers existed already at $75^{\circ} \mathrm{C}$. Analogous to an Ostwald ripening process, these more mobile molecules allowed for fusion of nanocrystals. After the sample was cooled back to room temperature (fig. 3e), all molten polymers recrystallized. Hence, during annealing for about one hour at $75^{\circ} \mathrm{C}$, the arrangement (morphology) of the crystalline material changed due a melting and re-crystallization process involving the detachment of polymers from one nanocrystal and attachment at another crystal surface. This process led to a coarsening of the

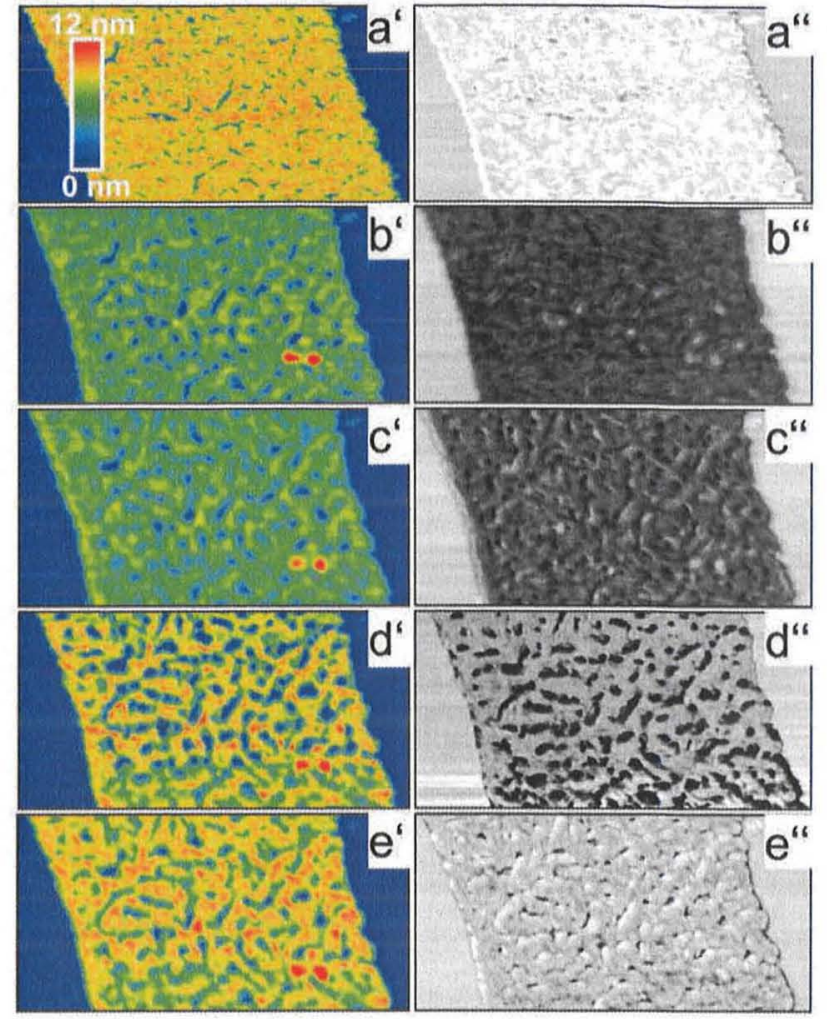

Fig. 3. (Colour on-line) AFM topography (left column, the color code ranges from 0 to $12 \mathrm{~nm}$ ) and phase (right column) images $\left(3 \times 1.5 \mu \mathrm{m}^{2}\right)$ showing reorganization within a monolayer of nanocrystals induced by annealing at $75^{\circ} \mathrm{C}$, i.e. ca. $60^{\circ} \mathrm{C}$ below the temperature where melting is detected by DSC. a) As prepared, b) $15 \mathrm{~min}$, c) $60 \mathrm{~min}$, d) $75 \mathrm{~min}$ after reaching the chosen temperature of $75^{\circ} \mathrm{C}$. Images e) show the sample after being quenched back to room temperature. The tapping oscillation amplitude and force were similar for all images except d) where much harder tapping conditions (lower amplitude and lower set point ratio) were applied.

morphology: Small crystals transformed into bigger aggregates. Interestingly, during this coarsening stage the thickness of the crystalline domains did not change significantly. Thus, diffusion of molecules between neighboring crystals and subsequent re-attachment/re-crystallization at crystal surfaces was probably so fast that polymers were not able to achieve less folded chain. We conclude that at such comparatively low temperatures with respect to the equilibrium melting temperature of ca. $141^{\circ} \mathrm{C}$ [43] the process is driven by the reduction of the number of molecules at crystal boundaries, i.e. a reduction in total perimeter of all crystals. This is achieved by making the crystalline domains bigger than the initial nanocrystals (fusion of small crystalline domains into bigger ones).

As can be seen in fig. 4, the rate of the coarsening process and the degree of coarsening was affected by the number of stacked layers of nanocrystals. At the edge of a multilayer stack one can observe a monolayer, a double layer and a triple layer simultaneously (fig. 4a). For 


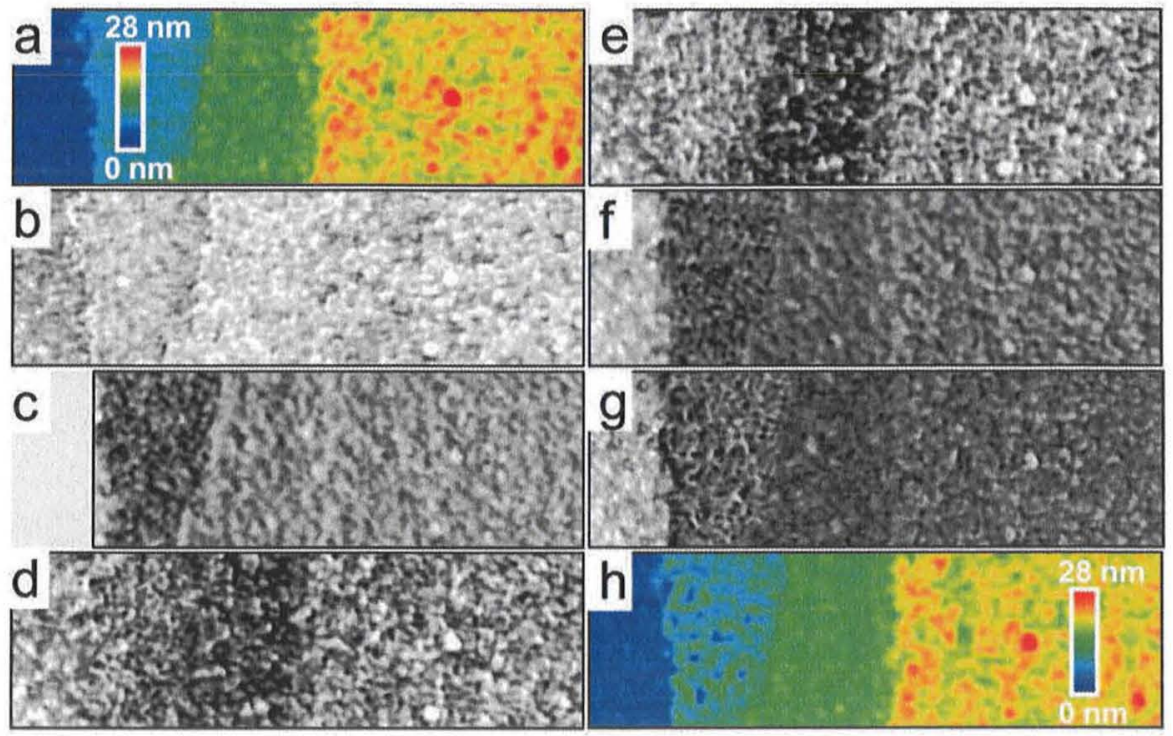

Fig. 4. (Colour on-line) AFM topography ( $\mathrm{a}+\mathrm{h}$, the color code ranges from 0 to $28 \mathrm{~nm})$ and phase images $(\mathrm{b}-\mathrm{g})\left(5 \times 1.5 \mu \mathrm{m}^{2}\right)$ showing the evolution of a stack of three layers of nanocrystals at $80^{\circ} \mathrm{C}$ (c) $\left.15 \mathrm{~min}, \mathrm{~d}\right) 60 \mathrm{~min}$, e) $330 \mathrm{~min}$ ) followed by annealing at $\left.100^{\circ} \mathrm{C}(\mathrm{f}) 15 \mathrm{~min}, \mathrm{~g}\right)+\mathrm{h}$ ) $\left.240 \mathrm{~min}\right)$. Images a) and b) show the as-prepared sample.

the as-prepared sample, the phase contrast in all these regions indicates rather elastic, i.e. crystalline material (fig. 4b). After raising the temperature to $80^{\circ} \mathrm{C}$ (fig. 4c), first the monolayer partially melted and started to reorganize. Only about one hour later, also the double layer showed clear indications of partial melting and rearrangements (fig. 4d). A few hours later, the monolayer has coarsened to such an extent that the fraction of molten polymers was so small that it was hardly detectable in the phase image while the region of the double layer contained still a significant amount of molten polymers (fig. 4e). The triple layer was still comparatively unaffected. These differences in annealing behavior of the various layers might be attributed to the change in interfacial energy between substrate and nanocrystal and between layers of nanocrystals. Zhang et al. [55] showed that layered lamellar crystals of short polyethylene molecules showed a sequential melting behavior: the top lamella melted before the bottom lamella, quantitatively related to stabilization via van der Waals interactions. In our experiments on layers of nanocrystals annealed at significantly lower temperatures rearrangement processes driven by detachment of molecules from the nanocrystals occurred first in the monolayer region, probably favored by a reduced areal density of polyethylene molecules.

At $100{ }^{\circ} \mathrm{C}$, the fraction of molten polymers increased homogeneously for all regions as can be deduced from a homogeneous "darkening" of the phase contrast. The most elastic (hardest) part of the image corresponds to the substrate, seen on the outmost left part of the image of fig. 4f. Even during annealing for several hours no significant further changes were detectable on the scale of the image (fig. 4g). Comparing the topography after this annealing procedure (fig. $4 \mathrm{~h}$ ) with the initial stage (fig. 4a) demonstrates that only local coarsening had occurred, crystals fused to larger object without change in the degree of chain folding. As the thickness of these layers has not changed, we suppose that no interdiffusion between layers had occurred.

In summary, we attribute the observation of significant changes at such low temperatures in polyethylene crystals mainly to the enormous number of polymers attached to crystal surfaces (i.e., lateral faces of lamellar crystal). These molecules have a low but still measurable probability to detach from the crystal and diffuse to other crystalline surfaces. Of course, the same process is also present in larger crystals but there the absolute number of molecules detaching/attaching per unit time is less and thus the resulting morphological changes are hardly detectable or would require significantly longer annealing times.

\subsection{Annealing experiments at $\mathrm{T}<120^{\circ} \mathrm{C}$}

Annealing behavior of solution grown polyethylene single crystals and investigations of morphological changes during annealing of such crystals have been studied previously by various groups [56-59]. In particular, upon annealing of single crystals placed on a solid substrate the formation of picture frame-like structures was studied in situ using AFM at elevated temperatures which indicated that the reorganization process started at the crystal edges at a temperature above ca. $120^{\circ} \mathrm{C}$. In our case, the thickness of the crystalline regions changed gradually but clearly when annealing at temperatures higher than ca. $100^{\circ} \mathrm{C}$ (fig. 5). While at the lower temperatures only reorganization without detectable changes in height occurred (fig. 5a), thickness started to increase at higher temperatures and isolated crystalline domains formed at around 

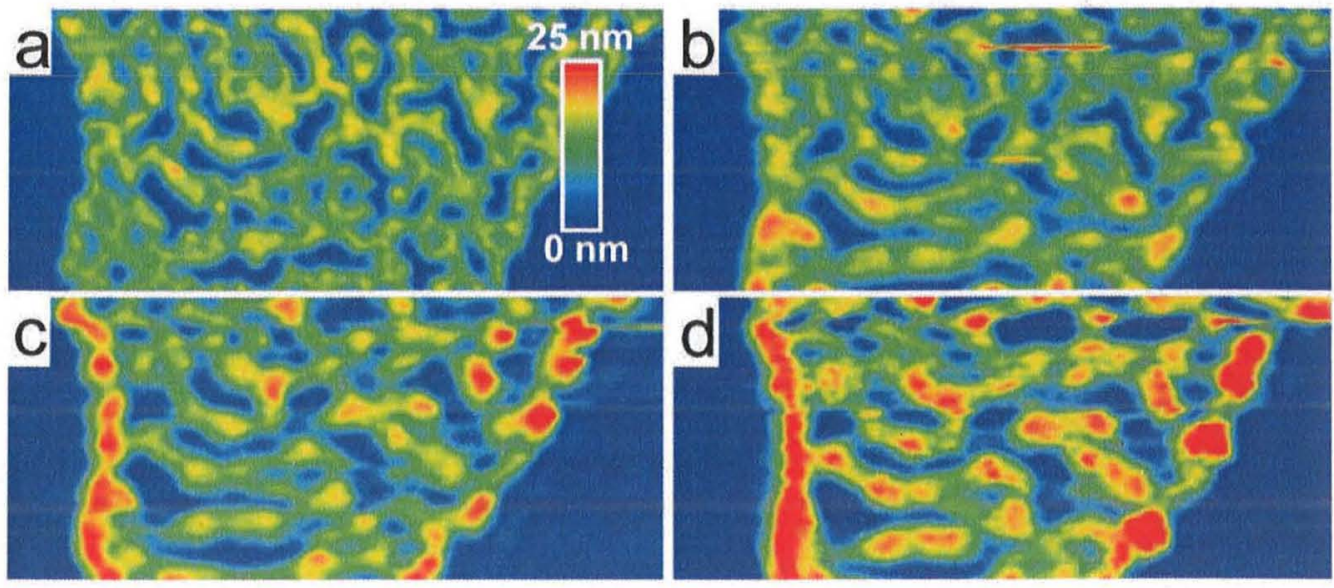

Fig. 5. (Colour on-line) AFM topography images $\left(3.5 \times 1.5 \mu \mathrm{m}^{2}\right)$ of a monolayer of nanocrystals, measured after short annealing times at progressively higher temperatures: a) after $30 \mathrm{~min}$ at $100^{\circ} \mathrm{C}$, b) after $15 \mathrm{~min}$ at $110^{\circ} \mathrm{C}, \mathrm{c}$ ) after $15 \mathrm{~min}$ at $117^{\circ} \mathrm{C}$, and d) after $20 \mathrm{~min}$ at $120^{\circ} \mathrm{C}$.

$110^{\circ} \mathrm{C}$ (fig. 5b). At even higher temperatures (fig. $5 \mathrm{c}+\mathrm{d}$ ), the process accelerated. From these images it is quite notable that the boundaries of the monolayer region thickened more rapidly than regions within the monolayer, analogous to what was observed for monolayer crystals of poly (ethylene oxide) $[52,53,60-62]$ or polyethylene [56$59]$. Based on these results, we conclude that at temperatures higher than ca. $110^{\circ} \mathrm{C}$ the reduction of chain fold became possible. The higher thermal energy increased the probability of chain desorption, allowed for faster rearrangements over larger distances. This favored the formation of isolated domains and, in turn, provided sufficient time for thickening before additional polymers reattached to the crystal front. Thickening was more prominent in regions with a lower amount of surrounding polymers, in particular at the boundaries of the initial monolayer stripe.

\subsection{Annealing experiments at $\mathrm{T}<130^{\circ} \mathrm{C}$}

When annealing a stack of three layers, additional features were observed (fig. 6). Similar to the monolayer of fig. $5 \mathrm{~d}$, after one hour at $122^{\circ} \mathrm{C}$ (fig. 6b), the monolayer part had reorganized into an array of almost individual crystalline domains with a thickness up to three times the initial thickness. Taking into account that the volume, i.e. the amount of molecules, of this monolayer region has not changed detectably, this implies that the polymers chains became less folded. Secondly and rather surprisingly, the triple-layer region became thinner, as thin as the doublelayer region. This implies that in the triple layer significant interdiffusion occurred between the layers of nanocrystals. After such annealing, the height of the initial triple layer was found to be the same as for the initial double layer. This suggests that the degree of chain folding is the same in both regions. The most prominent thickness was around $16 \mathrm{~nm}$, i.e. twice the starting thickness, which can be interpreted as a reduction in the number of chain folds by a
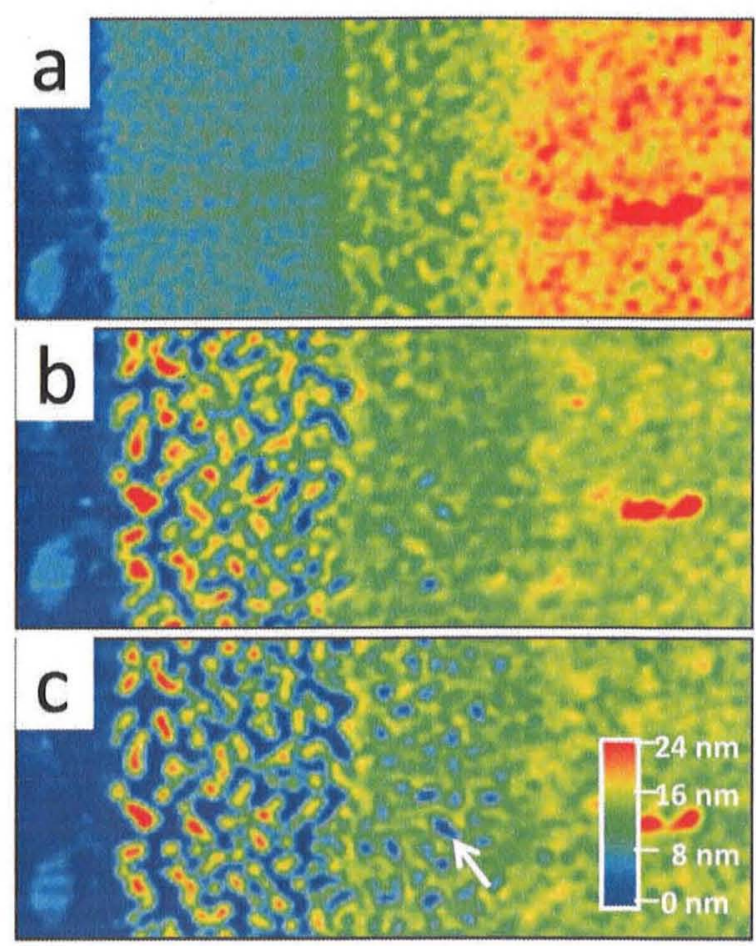

Fig. 6. (Colour on-line) AFM topography images $\left(5 \times 2 \mu \mathrm{m}^{2}\right)$ of a stack of three layers of nanocrystals, all measured at room temperature. a) As prepared, b) after 1 hour at $122^{\circ} \mathrm{C}$, and c) after 1 hour at $124^{\circ} \mathrm{C}$. The monolayer reorganized faster than the double and triple layer. The three layers merged and resulted in a layer having about the thickness of a double layer. The double layer visible reorganized before the triple layer. In the monolayer region isolated crystalline islands started to form. The height of some of these islands increased even beyond the thickness of the double layer. 


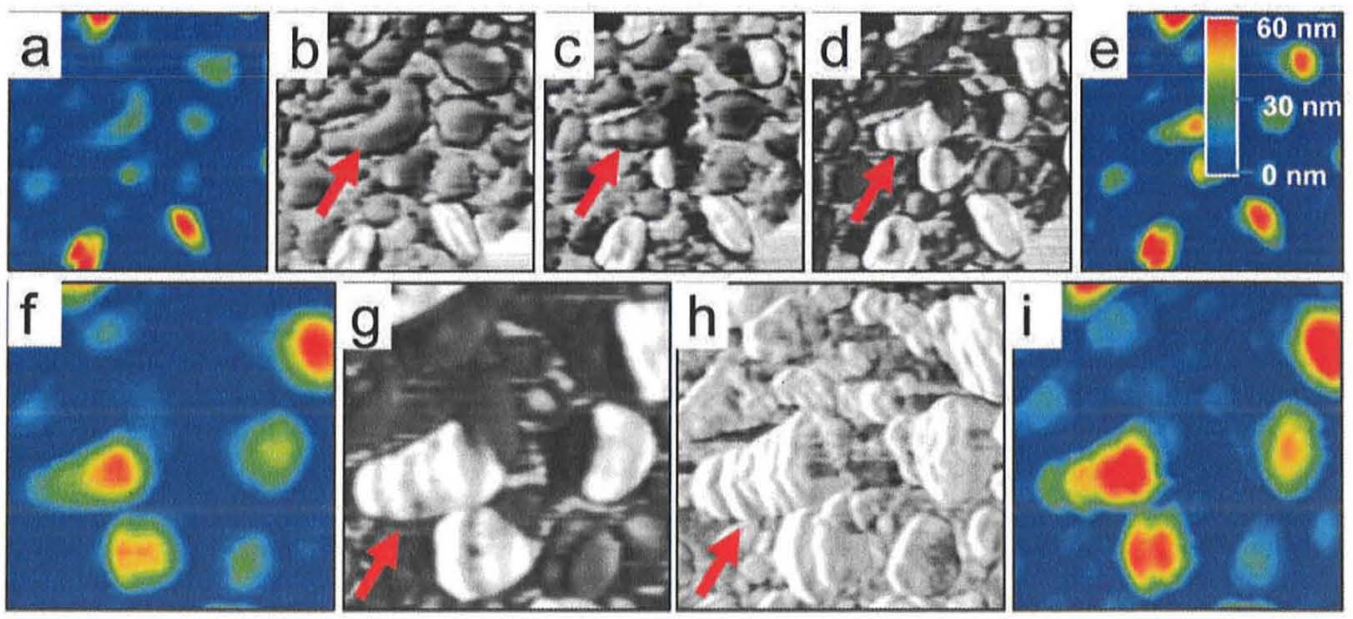

Fig. 7. (Colour on-line) AFM topography (a), e), f), and i), color code ranging from 0 to $60 \mathrm{~nm}$ ) and phase (b), c), d), g) and h)) images of a monolayer of nanocrystals pre-annealed at $110^{\circ} \mathrm{C}$ for $30 \mathrm{~min}$ and then measured during annealing at $120^{\circ} \mathrm{C}$ (a) and b): $15 \mathrm{~min}, \mathrm{c}$ ): $55 \mathrm{~min}, \mathrm{~d}$ ) and e): $120 \mathrm{~min}, \mathrm{f}$ ) and g): $190 \mathrm{~min}$ ) and after quenching to at room temperature (h) and i)). The red arrow highlights one droplet-like region which melted as the temperature was raised from $110^{\circ} \mathrm{C}$ to $120^{\circ} \mathrm{C}$ but then re-crystallized during annealing at $120^{\circ} \mathrm{C}$. In the course of annealing, progressively more of these droplet-like regions re-crystallize. The arrows are used to highlight one of these droplets which clearly underwent crystallization in the course of annealing. The size of images a)-e) is $1.8 \times 1.8 \mu \mathrm{m}^{2}$ and of images $\mathrm{f}$ ) $-\mathrm{i}$ ) is $1.2 \times 1.2 \mu \mathrm{m}^{2}$.

factor of two. Taking into account that we have observed significant melting-recrystallization taking place even at lower temperatures, we tentatively conclude that the double layer region has transformed into a monolayer of crystalline domains having more extended chains. Along these arguments, the reduction in thickness of the triple layer can be interpreted also by the formation of a monolayer of thicker crystalline domains. However, as more nanocrystals (roughly 1.5 times the number of nanocrystals deposited in the double layer region) were available at the beginning of annealing, the resulting coarsened crystalline domains were less separated. This conclusion is supported by the formation of comparatively large empty regions within this double layer region, which did not appear in the triple layer region, when the sample was further annealed at $124^{\circ} \mathrm{C}$ for one hour. One of these spots is indicated by an arrow in fig. 6c. It is highly intriguing to observe that for a given annealing time thickening was the more significant the fewer molecules were surrounding the crystalline domain. This is the case at the periphery of the initial monolayer (fig. 5d) and within the monolayer (fig. 6). In regions with a higher amount of molecules per area, the attachment probability is higher, the time between successive attachments is shorter and thus the available time is shorter for molecules at the crystal boundaries to remove chain folds. The lateral faces of the crystals are identified as a key element during annealing and, in particular, in the coarsening process. It is at these faces where molecules attach and detach. Thus, for small nanocrystals the number of molecules at these surfaces is significant with respect to the total number of molecules in the crystal. Following these fundamental arguments which lead to the Gibbs-Thomson relation, we thus expect that the surface to volume ratio of small nanocrystals is not only determined by the thickness of the lamellar crystal (the thinner the lamella the higher is the surface to volume ratio) but also by the lateral size. In addition, the strength of attachment of molecules at the various faces of the crystal may be different (as it is the case for polyethylene crystals) and consequently one has to "weigh" the contributions of the different faces not only according to their area but also as a function of their attachment strength (=surface tension) which is also a function of temperature. As our results are not providing all the necessary details for an in-depth analysis with respect to the contributions of the individual faces we are only able to discuss this point in general terms.

At $120^{\circ} \mathrm{C}$, the coarsening process in a monolayer region was followed in time and in situ in fig. 7. Already after $15 \mathrm{~min}$, the monolayer had transformed into an array of droplet-like domains of a variety of sizes (fig. 7a). Interestingly, the corresponding phase image clearly indicates that most of these droplets exhibited a liquid-like behavior, at least at their surface (the chosen conditions of tapping mode AFM, i.e. the set-point ratio [54,63], allowed only for small penetration of a few nanometers). Initially, only very few droplets contained detectable crystals. Interestingly, in the course of annealing over several hours (keeping the conditions of tapping mode AFM constant), more of these liquid-like droplets crystallized and grew in size (fig. 7c-g). The thickness of the crystalline domains reached values of up to ca. $60 \mathrm{~nm}$. The layered topography pattern, together with the internal structure of the crystalline domains is visible in the phase image. The data presented in fig. 7 and results from many other samples consisting of droplet-like regions strongly indicate that the droplets contain stacks of lamellae. From AFM it is not possible to decide definitely if these lamel- 

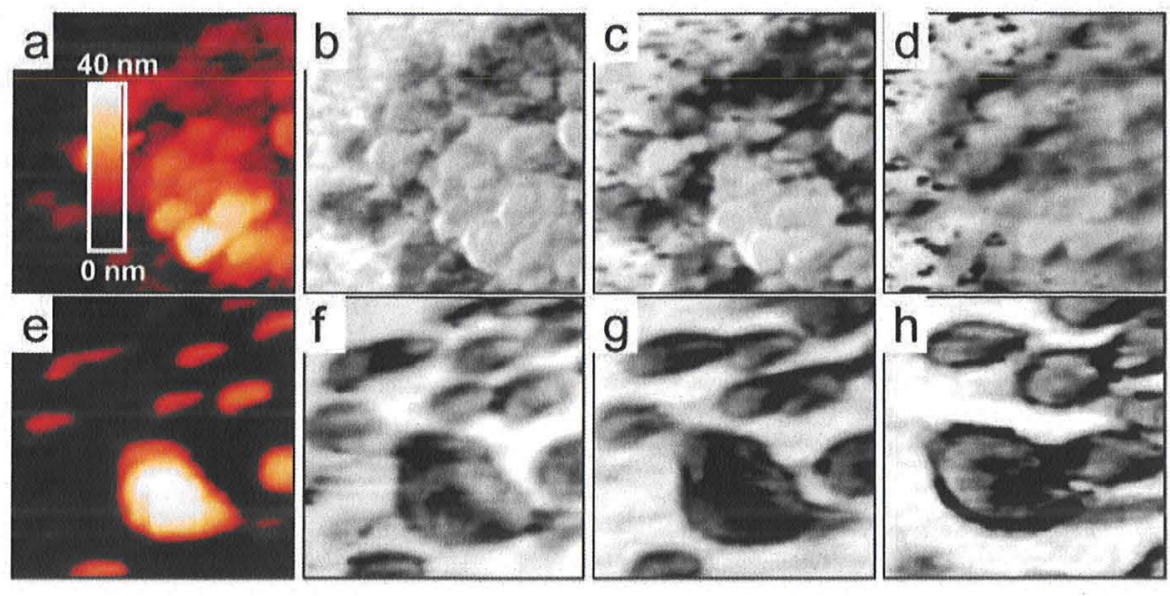

Fig. 8. (Colour on-line) AFM topography (a) and e), color code ranging from 0 to $40 \mathrm{~nm}$ ) and phase (b)-d), f-h)) of a disordered pile of nanocrystals. a) + b): As prepared, c) after $15 \mathrm{~min}$ at $75^{\circ} \mathrm{C}$, and after each time additional $15 \mathrm{~min}$ at d) $100^{\circ} \mathrm{C}$, e $+\mathrm{f}$ ) $\left.120^{\circ} \mathrm{C}, \mathrm{g}\right) 126^{\circ} \mathrm{C}$, and h) $133^{\circ} \mathrm{C}$. The size of images is $1 \times 1 \mu \mathrm{m}^{2}$.

lae are fully edge-on or somewhat inclined. E.g., we cannot exclude that the lamellae are tilted by ca. $35^{\circ}-45^{\circ}$, which would suggest that the stems are parallel to the substrate but lamellae are inclined.

After cooling the sample back to room temperature, the phase image (fig. $7 \mathrm{~h}$ ) demonstrates that all the molten polymers crystallized. The corresponding topography image (fig. 7i) clearly shows that the thickness of the resulting new crystalline domains was mostly only around $10 \mathrm{~nm}$ (see the differences between fig. $7 \mathrm{f}$ and fig. 7i), i.e. a thickness consistent with the lamellar thickness obtained when annealing the sample at temperatures below ca. $110^{\circ} \mathrm{C}$.

\subsection{Annealing experiments up to $\mathrm{T}=133^{\circ} \mathrm{C}$}

In order to follow the reorganization process at comparatively high annealing temperatures, we decided to use a small pile of a limited number of nanocrystals which allowed observing simultaneously the evolution of regions of different thickness. The focus was on which part of this pile "survives" the annealing process at a given temperature. We would like to emphasize that such a pile of a limited number of nanocrystals sitting on a bare substrate has to be considered as an "open system" as polymer chains have the possibility to diffuse away from the pile once they are detached from the nanocrystals. This has to be contrasted to the "closed system" of nanocrystals dispersed in an aqueous medium used by Rochette et al. [9]. There, no exchange of polymers between nanocrystals is likely to occur. Thus, equilibration of nanocrystals at various temperatures is feasible.

In the present experiments, we kept the annealing time as short as possible for each temperature chosen. Under such conditions, we did not allow for long-time rearrangement and coarsening processes. The equipment used required a minimum of ca. $15 \mathrm{~min}$ to stabilize the temperature and to obtain a suitable AFM image. As shown already for nanocrystals deposited in stripes, annealing at temperatures up to ca. $110{ }^{\circ} \mathrm{C}$ allowed only rearrangement processes on small length-scales. It can be seen (fig. 8c) that in comparison to thicker parts the thinner parts of the sample exhibited a more liquid-like behavior. Above ca. $110^{\circ} \mathrm{C}$ significant coarsening occurred and resulted in very few crystals of different sizes. The biggest crystal evolved from the thickest region of the initial pile. Increasing the annealing temperature step-wise (figs. $8 \mathrm{f}-\mathrm{g}$ ) led to fusion of the smaller crystals and to some increase in size of the bigger ones. As indicated by the pronounced contrast in the phase images of fig. 8, at elevated temperatures all crystals were surrounded and covered by molten polymers. Thicker crystals were able to attract more of these mobile molecules from the thinner ones and thus got larger in the course of the measurement. On the contrary, smaller (and thinner) crystals became even smaller by losing their molecules to the thicker crystals. This observation is in accordance with a theoretical study by Rabani et al. who concluded that thermal coarsening of nanoparticle domains proceeds via diffusion and coalescence of domains [15].

Besides the number of chain folds per polymer, the thickness of crystalline polyethylene lamellae depends also on the degree of chain tilting within the crystal. However, based on AFM measurements alone, we are not able to determine the tilt angle. Previous works e.g. for long alkanes [64] have shown that for lamellae crystallized at temperatures lower than ca. $100^{\circ} \mathrm{C}$ chain tilting is less pronounced than upon annealing at temperatures closer to the melting point. For fully extended alkanes of a total length of about $30 \mathrm{~nm}$ the tilt angle increased from $0^{\circ}$ to about $40^{\circ}$ upon heating from ca. $100^{\circ} \mathrm{C}$ to ca. $125^{\circ} \mathrm{C}$. By means of clectron diffraction Labaig et al. [65] observed for crystals grown on a supporting substrate at temperatures as high as $\sim 125-131^{\circ} \mathrm{C}$ chain tilt of $\sim 45^{\circ}$ (cited e.g. by Keith et al. [66]). Thus, we may assume that also in our (mono) lamellar crystals chain tilt will increase as we increase the annealing temperature. 


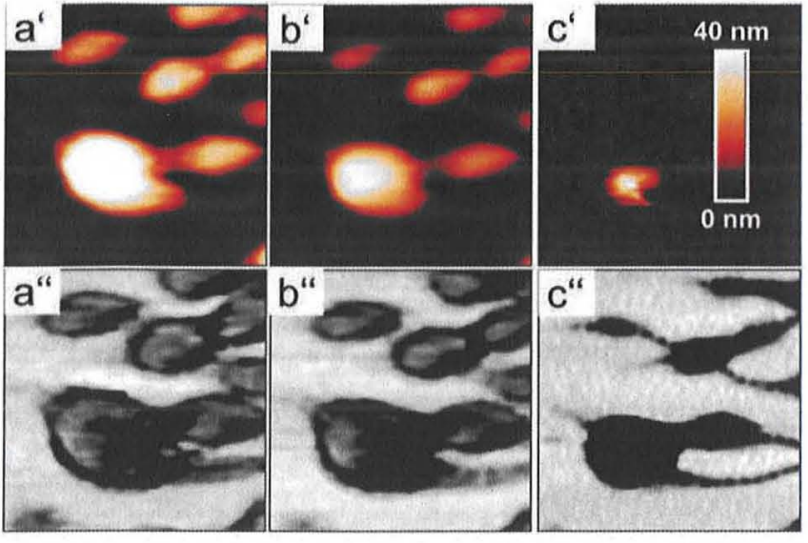

Fig. 9. (Colour on-line) AFM topography (top row, color code ranging from 0 to $40 \mathrm{~nm}$ ) and phase (bottom row) images showing the disappearance in time of the nanocrystals from fig. $8 \mathrm{~h}$ after a) $15 \mathrm{~min}$, b) $300 \mathrm{~min}$ and c) $1000 \mathrm{~min}$ at $133^{\circ} \mathrm{C}$. The size of images is $1 \times 1 \mu \mathrm{m}^{2}$.

This coarsening process at temperatures between $120^{\circ} \mathrm{C}$ and $133^{\circ} \mathrm{C}$ is associated with a size-dependent melting temperature: at a comparatively low temperature the smaller crystals started to melt and disappeared eventually while at this temperature bigger crystals still increased in thickness. In order to explore the influence of annealing time we kept the sample of fig. 8 at a constant annealing temperature of $T_{A}=133^{\circ} \mathrm{C}$ (fig. 9). The choice of $T_{A}$ was inspired by the position of the melting peak observed in DSC. Comparing fig. 8e with fig. 9a shows that the step-wise increase of $T_{A}$ from $120^{\circ} \mathrm{C}$ to $133^{\circ} \mathrm{C}$ did not lead to melting of all crystals. Only some of the small crystals melted and fused. On the contrary, the biggest crystal slightly increased in size.

Furthermore, from a comparison of the total volume of the all objects, either crystalline or molten, we conclude that after such short annealing times the amount of polymeric material stayed approximately constant. However, keeping the sample at $133^{\circ} \mathrm{C}$ for many hours eventually led to the disappearance of all crystals (figs. 9b and c). After ca. $1000 \mathrm{~min}$ at $133^{\circ} \mathrm{C}$ (fig. 9c), one hardly can detect any crystalline domain. The AFM phase contrast image suggests that all crystals have been transformed into thin liquid droplets. From the topography image, it is obvious that the volume of the crystalline domains was not conserved. Taking into account that polymers cannot evaporate, a significant number of polymers must have diffused out of the frame of the image. These polymers probably coat the surrounding substrate with a layer too thin to be detectable by AFM (at the measurement parameters chosen). We interpret this disappearance of crystals in the following way: Molecules at the surface of any crystal have a non-zero probability to detach from this crystal, e.g. sublimation of ice below the melting point. At temperatures largely below the melting temperature, the detachment probability is low and almost no molecule would detach from a crystal within a given time, say, several hours. However, at temperatures close

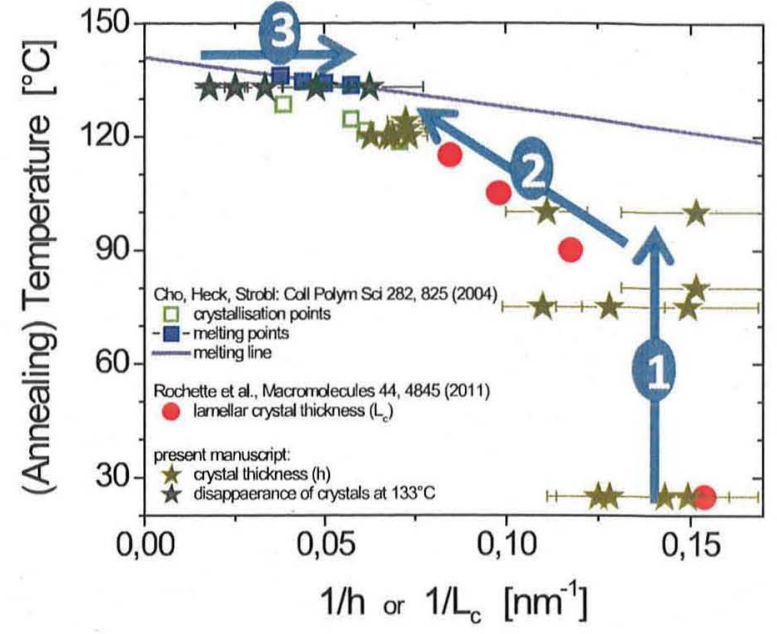

Fig. 10. Compilation of the thickness values determined from AFM measurements on monolayers shown in this manuscript, represented in a Gibbs-Thompson plot (temperature vs. reciprocal thickness). Our results are compared with literature data on bulk samples and on nanocrystals in aqueous dispersion analogous to the ones studied here on a solid support. The arrows 1-3 indicate pathways followed during annealing. Details are described in the text.

to the melting point, the detachment probability can be quite significant. As the crystals were deposited on an initially bare substrate they were surrounded by a large surface which does not contain any polymers (no free polymers were adsorbed onto the substrate because only nanocrystals were deposited). Thus, some of the detached molten polymers gained translational entropy by diffusing away from the crystal and so covered the initially bare substrate. This diffusion process away from the crystals progressively lowered the probability for re-attachment as the density of detached molecules close to the crystal decreased. Consequently, crystals progressively lost more and more molecules, got smaller and eventually reached a size where the surface-to-volume ratio was high enough to allow for complete melting of the crystal. Accordingly, small isolated crystals on any bare substrates can never "survive" for long times, they will eventually disappear as can been seen in fig. 9c. A related study for alkane monolayers and sub-monolayers showed the dependence of the melting temperature on surface coverage. There, crystals have been in equilibrium with a thin liquid layer covering the whole substrate [67]. We conclude that isolated crystals deposited on a bare substrate are prone to disappear even at temperatures well below the melting temperature of such crystals surrounded by its melt.

\subsection{Three distinct paths for changes in morphology}

From the above results we can identify three processes for lowering the free energy of an ensemble of nanocrystals (see fig. 10). Annealing nanocrystals at progressively higher temperatures allows accentuating them differently. 
Path 1: Due to their small size, their high surface-tovolume ratio, a large amount of molecules at the periphery of these nanocrystals shows a high tendency for detachment. This favors the formation of bigger lamellar crystals at the expense of smaller ones. Interestingly, this Ostwald ripening process is not necessarily involving a reduction of the number of chain fold. i.e., the thickness of the lamellar crystals remains constant. Thus, we may tentatively conclude that the required energy for removing chain folds is higher than for detaching polymers from a crystal. Alternatively, the kinetics of fold removal may be slower in comparison to the exchange of molecules between individual nanocrystals.

Path 2: The existence of chain folds in an otherwise crystalline polymer reduces crystallinity and thus represents a cost in crystallization energy. Thus, states of highly folded crystalline chains are never stable and will evolve into less folded states, i.e. into lamellar crystals of higher thickness. Such can either be achieved at constant temperature by increasing the annealing time (the increase in lamellar thickness depends on the logarithm of annealing time $[68,69]$ ) or for a constant experimental (annealing) time by increasing the annealing temperature. Due to the high mobility of polyethylene along the chain axis lamellar thickening is easily possible even in the crystalline state [70-72]. In accordance with this tendency, our observations show an increase in lamellar thickness at temperatures above ca. $110^{\circ} \mathrm{C}$. We expect that some increase of lamellar thickness would also occur at lower temperatures but at a drastically lower rate.

Path 3: For our "open system", even below the thermodynamic equilibrium melting temperature crystals can show a melting-like behavior. Similar to sublimation, bordered by empty space or a bare substrate, crystals of small volume (i.e., with a large number of molecules located at the crystal surface) will decrease in size due to the loss of molecules to the surrounding space. We would like to re-emphasize that such a decrease in crystal volume and eventual melting is not possible in a "closed system" like the one studied by Rochette et al. [9].

\section{Concluding remarks}

Taking advantage of the well-defined small size of the initial nanocrystals, which were deposited in a controlled way to result in a layered assembly, we investigated the process of molecular reorganization and the corresponding morphological changes as a function of temperature and annealing time. Due to the kinetic features of the various pathways taken, a representation of the reciprocal crystal thickness in a Gibbs-Thompson plot yields a complex pattern. A large spectrum of possible correlations between annealing temperature and crystal thickness is explored, as shown in fig. 10. No crystal will be able to resist melting if the temperature is increased above the melting line which reflects the size-dependent melting temperature [43]. We note that the representation as a function of inverse thickness implies that the crystal size in the other two dimensions is large. Thus, for the initial nanocrystals having a nanometer size in all dimensions, a representation as a function of inverse volume might be more appropriate. Below the melting line, the actual thickness of the crystal depends on the pathway taken, allowing to obtain for a given annealing temperature crystals of different lamellar thickness.

A comparison with literature results on bulk samples [42] and results obtained by annealing the same type of polyethylene nanocrystals in an aqueous dispersion [9] demonstrates that our results are consistent with previous conclusions. As shown in [9] for a "closed system", crystals having a well-defined lamellar thickness can be stabilized (equilibrated) at temperatures well below the melting line for large lamellar crystals of a certain lamellar thickness, as studied e.g. in [42]. Furthermore, by using assemblies of small nanocrystals ("open system"), we were able to display that polymer crystals are thermodynamically not stable below the melting line. While such crystals will always try to reach energetically more favorable states of less folded chains, i.e. increased lamellar thickness, they may also lose molecules to the surrounding bare substrate, decrease in volume and eventually melt due to their small size. Consequently, a manifold of crystalline states, differing e.g. in lamellar thickness and volume, are possible for a given annealing temperature. In an "open system" these states are only metastable. Thus, morphological changes will occur in the course of time. The molecular architecture of polyethylene allows for comparatively fast reorganization processes and a relatively facile thickening of lamellar crystals which can be easily detected even after rather short time and modest annealing temperatures. We anticipate that also other semicrystalline polymers will follow the same pathways during annealing. However, steric constraints or specific intra- or intermolecular interaction may drastically reduce the rate of these kinetic processes. Thus, on typical experimental time scales no changes in thickness or morphology may be detectable.

This research was supported by Baden-Württemberg Stiftung in the framework of the Kompetenznetz Funktionelle Nanostrukturen (project A5). We wish to thank Matthias Ballauff and Gert Strobl for critical reading of the manuscript and helpful comments.

\section{References}

1. P. Hanarp, D.S. Sutherland, J. Gold, B. Kasemo, Colloids Surf. A: Physicochem. Engin. Aspects 214, 23 (2003).

2. J. Schmitt, G. Decher, W.J. Dressick, S.L. Brandow, R.E. Geer., R. Shashidhar, J.M. Calvert, Adv. Mater. 9, 61 (1997).

3. R. Shenhar, E. Jeoung, S. Srivastava, T. Norsten, V. Rotello, Adv. Mater. 17, 2206 (2005).

4. H. Bu, Y. Pang, D. Song, T. Yu, T.M. Voll, G. Czornyj, B. Wunderlich, J. Polym. Sci. Part B: Polym. Phys. 29, 139 (1991).

5. H. Bu, J. Cao, Z. Zhang, Z. Zhang, R. Festag, D.C. Joy, Y.K. Kwon, B. Wunderlich, J. Polym. Sci. Part B: Polym. Phys. 36, 105 (1998). 
6. Q. Tong, M. Krumova, S. Mecking, Angew. Chem. Int. Ed. 47, 1 (2008).

7. Q. Tong, M. Krumova, I. Göttker-Schnetmann, S. Mecking, Langmuir 24, 2341 (2008).

8. C.H.M. Weber, A. Chiche, G. Krausch, S. Rosenfeldt, M. Ballauff, L. Harnau, I. Göttker-Schnetmann, Q. Tong, S. Mecking, Nano Lett. 7, 2024 (2007).

9. C.N. Rochette, S. Rosenfeldt, K. Henzler, F. Polzer M. Ballauff, Q. Tong, S. Mecking, M. Drechsler, T. Narayanan, L. Harnau, Macromolecules 44, 4845 (2011).

10. J.E. O'Gara, J.D. Portmess, K.B. Wagener, Macromolecules 26, 2837 (1993).

11. K.B. Wagener, D. Valenti, S.F. Hahn, Macromolecules 30 , 6688 (1997).

12. H. Yabu, M. Shimomura, Adv. Funct. Mater. 15, 575 (2005).

13. M. Ghosh, F. Fan, K.J. Stebe, Langmuir 23, 2180 (2007).

14. L.V. Govor, G. Reiter, J. Parisi, G.H. Bauer, Phys. Rev. E 69, 061609 (2004).

15. E. Rabani, D.R. Reichman, P.L. Geissler, L.E. Brus, Nature 426, 271 (2003)

16. E. Pauliac-Vaujour, P. Moriarty, J. Phys. Chem. C 111, 16255 (2007).

17. C.J. Murphy, T.K. Sau, A.M. Gole, C.J. Orendorff, J. Gao, L. Gou, S.E. Hunyadi, T. Li, J. Phys. Chem. B 109, 13857 (2005).

18. T.K. Sau, C.J. Murphy, Langmuir 21, 2923 (2005).

19. J. Xu, J. Xia, S.W. Hong, Z. Lin, F. Qiu, Y. Yang, Phys. Rev. Lett. 96, 066104 (2006)

20. S. Hong, J. Xia, Z. Lin, Adv. Mater. 19, 1413 (2007).

21. E. Adachi, A.S. Dimitrov, K. Nagayama, Langmuir 11, 1057 (1995)

22. R.D. Deegan, O. Bakajin, T.F. Dupont, G. Huber, S.R. Nagel, T.A. Witten, Nature 389, 827 (1997)

23. O. Karthaus, L. Gracjo, N. Maruyama, M. Shimomura, Chaos 9, 308 (1999).

24. R.D. Deegan, Phys. Rev. E 61, 475 (2000)

25. R.D. Deegan, O. Bakajin, T.F. Dupont, G. Huber, S.R. Nagel, T.A. Witten, Phys. Rev. E 62, 756 (2000).

26. H. Hu, R.G. Larson, J. Phys. Chem. B 110, 7090 (2006).

27. G. Strobl, The Physics of Polymer, 3rd edition (Springer, Berlin, 2007).

28. A. Keller, Philos. Mag. 2, 1171 (1957).

29. E.W.Z. Fischer, Naturforsch. A 12, 753 (1957).

30. A. Keller, A. O'Connor, Nature 180, 1289 (1957).

31. Z. Bartczak, A.S. Argon, R.E. Cohen, T. Kowalewski, Polymer 40, 2367 (1999)

32. O. Mellbring, S. Kihlman Oiseth, A. Krozer, J. Lausmaa, T. Hjertberg, Macromolecules 34, 7496 (2001).

33. Y. Wang, S. Ge, M. Rafailovich, J. Sokolov, Y. Zou, H. Ade, J. Lüning, A. Lustiger, G. Maron, Macromolecules 37, 3319 (2004).

34. J.U. Sommer, Eur. Phys. J. E 19, 413 (2006).

35. S. Rastogi, Macromolecules 30, 7880 (1997).

36. M. Hikosaka, K. Amano, S. Rastogi, A. Keller, J. Mater. Sci. 35, 5157 (2000).

37. D.I. Bower, An Introduction to Polymer Physics (Cambridge University Press, 2002).
38. M. Schmidt, R. Kusche, B.V. Issendorff, H. Haberland, Nature 393, 238 (1998).

39. P.W. Voorhees, J. Stat. Phys. 38, 231 (1985).

40. W. Ostwald, Phys. Chem. 22, 289 (1897)

41. N. Metatla, S. Palato, B. Commarieu, J.P. Claverie, A Soldera, Soft Matter 8, 347 (2012).

42. B. Heck, G. Strobl, T.Y. Cho, Colloid Polym. Sci. 282 825 (2004)

43. G. Strobl, Eur. Phys. J. E 18, 295 (2005).

44. T.A. Witten, EPL 86, 64002 (2009).

45. A. Dong, J. Chen, P.M. Vora, J.M. Kikkawa, C.B. Murray, Nature 466, 474 (2010).

46. C.P. Martin, M.O. Blunt, P. Moriarty, Nano Lett. 4, 2389 (2004).

47. C.P. Martin, M.O. Blunt, E. Pauliac-Vaujour, A. Stannard, P. Moriarty, Phys. Rev. Lett. 99, 116103 (2007).

48. Q. Tong, S. Mecking, J. Polym. Sci. Part A: Polym. Chem. 47, 6420 (2009).

49. L. Frastia, A.J. Archer, U. Thiele, Phys. Rev. Lett. 106 077801 (2011)

50. Y.O. Popov, T.A. Witten, Phys. Rev. E 68, 036306 (2003)

51. F. Zhang, J. Liu, H. Huang, B. Du, T. He, Eur. Phys. J E 8, 289 (2002)

52. J.-U. Sommer, G. Reiter, Europhys. Lett. 56, 755 (2001)

53. G. Reiter, G. Castelein, J.-U. Sommer, Phys. Rev. Lett. 8 5918 (2001).

54. G. Bar, Y. Thomann, R. Brandsch, H.-J. Cantow, Langmuir 13, 3807 (1997)

55. F. Zhang, G.G. Baralia, B. Nysten, A.M. Jonas, Macromolecules 44, 7752 (2011)

56. M. Tian, J. Loos, J. Polym. Sci. Part B: Polym. Phys. 39 $763(2001)$

57. J. Loos, M. Tian, Polymer 47, 5574 (2006)

58. S.N. Magonov, N.A. Yerina, G. Ungar, D.H. Reneker, D.A Ivanov, Macromolecules 36, 5637 (2003).

59. S.J. Organ, J.K. Hobbs, M.J. Miles, Macromolecules 37 , $4562(2004)$.

60. J.-U. Sommer, G. Reiter, Thermochim. Acta 432, 135 (1967).

61. Y.-X. Liu, E.-Q. Chen, Coord. Chem. Rev. 254, 1011 (2010).

62. D.-S. Zhu, Y.-X. Liu, A.-C. Shi, E.-Q. Chen, Polymer 47, 5239 (2006).

63. A. Knoll, R. Magerle, G. Krausch, Macromolecules 34 4159 (2001).

64. D.S.M. de Silva, X.B. Zeng, G. Ungar, S.J. Spells, Macromolecules 35, 7730 (2002).

65. J.J. Labaig, Doctoral dissertation, Strasbourg (1978).

66. H.D. Keith, F.J. Padden, B. Lotz, J.C. Wittmann, Macromolecules 22, 2230 (1989).

67. H. Riegler, R. Köhler, Nat. Phys. 3, 890 (2007).

68. E.W. Fischer, Kolloid-Z. Z. Polym. 231, 458 (1967).

69. C. Luo, J.-U. Sommer, Macromolecules 44, 1523 (2011).

70. E.W.Z. Fischer, Macromolecules 41, 2514 (1968).

71. Y.-F. Yao, R. Graf, H.W. Spiess, S. Rastogi, Macromolecules 41, 2514 (2008).

72. T. Yamamoto, J. Chem. Phys. 129, 184903 (2008). 